\title{
Hemorragia Peri-Intraventricular Grave em Prematuros: Impacto na Mortalidade e no Neurodesenvolvimento aos 24 Meses
}

\author{
Survival and Neurodevelopmental Outcomes of Premature \\ Infants with Severe Peri-Intraventricular Hemorrhage at 24 \\ Months of Age
}

\author{
Joana AMARAL ${ }^{1}$, Sara PEIXOTO ${ }^{1}$, Dolores FARIA ${ }^{1}$, Cristina RESENDE ${ }^{1}$, Adelaide TABORDA $\triangle^{1}$ \\ Acta Med Port 2022 Jan;35(1):42-50 - https://doi.org/10.20344/amp.12295
}

\section{RESUMO}

Introdução: A hemorragia peri-intraventricular grave tem sido associada a maior mortalidade e sequelas do neurodesenvolvimento. Mantém-se controverso o impacto da hemorragia peri-intraventricular isolada, sem lesão da substância branca. O objetivo deste trabalho foi avaliar a influência da hemorragia peri-intraventricular grave, associada ou não a leucomalácia peri-ventricular quística, na mortalidade e no neurodesenvolvimento aos 24 meses.

Material e Métodos: Estudo de coorte retrospetiva que incluiu os recém-nascidos com hemorragia peri-intraventricular grave, internados numa maternidade de apoio perinatal diferenciado, entre 2006 e 2015, e dois controlos com a mesma idade gestacional, internados logo a seguir ao caso, sem hemorragia peri-intraventricular. A avaliação do neurodesenvolvimento, aos 24 meses, foi realizada em 99 crianças, com recurso à escala The Schedule of Growing Skills Scale I/ em 52 e à escala de desenvolvimento mental de Ruth Griffiths em 47 crianças. Considerou-se défice grave do neurodesenvolvimento: paralisia cerebral, atraso do desenvolvimento psicomotor, surdez com necessidade de prótese auditiva ou cegueira.

Resultados: Foram incluídos 41 recém-nascidos com hemorragia peri-intraventricular grave e 82 controlos. Ocorreram 23 óbitos, 16 $(39,0 \%)$ nas hemorragias peri-intraventricular graves e sete $(8,5 \%)$ nos controlos (OR 7,6; IC 95\% 2,6 - 20,4; $p<0,001)$. Verificou-se défice grave do neurodesenvolvimento em sete $(30,4 \%)$ no grupo de hemorragia peri-intraventricular grave e um $(1,3 \%)$ no grupo de controlos (OR 32; IC 95\% 3,7 - 281; $p<0,001$ ). Na análise individualizada, a mortalidade foi superior quer nas hemorragia peri-intraventricular grau III com leucomalácia peri-ventricular quística associada (OR 4,4 IC 95\% 1,3 - 14,2; $p=0,015$ ), quer na hemorragia peri-intraventricular grau IV (OR 12; IC 95\% 3,5 - 41,2; $p<0,001)$, em relação aos controlos. Verificaram-se também diferenças no défice grave do neurodesenvolvimento em relação aos controlos $(1,3 \%)$ na hemorragia peri-intraventricular grau III com leucomalácia peri-ventricular quística associada $(75,0 \%, p<0,001)$ e na hemorragia peri-intraventricular grau IV $(50,0 \%, p<0,001)$.

Conclusão: Os recém-nascidos com hemorragia peri-intraventricular de grau IV ou grau III com leucomalácia peri-ventricular quística associaram-se a maior mortalidade e sequelas graves do neurodesenvolvimento.

Palavras-chave: Cérebro/diagnóstico por imagem; Deficiências do Desenvolvimento/diagnóstico por imagem; Hemorragia Cerebral Intraventricular; Recém-Nascido de Muito Baixo Peso

\section{ABSTRACT}

Introduction: Severe peri-intraventricular haemorrhage has been associated with higher mortality and neurodevelopmental impairment. The impact of peri-intraventricular haemorrhage alone (without white matter injury) remains controversial. The aim of this study was to evaluate the influence of severe peri-intraventricular haemorrhage, associated or not with cystic peri-ventricular leukomalacia, on mortality and neurodevelopment at 24 months.

Material and Methods: Retrospective cohort study, that included newborns with severe peri-intraventricular haemorrhage admitted to a maternity hospital with differentiated perinatal support between 2006 and 2015, and two controls with the same gestational age, without peri-intraventricular haemorrhage, who were admitted immediately after the case. Neurodevelopmental assessment, at 24 months, was performed in 99 children, using the Schedule of Growing Skills II scale in 52 and the Ruth Griffiths mental development scale in 47 children. Severe neurodevelopmental deficit was diagnosed in the following conditions: cerebral palsy, delayed psychomotor development, deafness requiring hearing aids and blindness.

Results: The study included 41 cases and 82 controls. Out of these, 23 died, 16 (39.0\%) in the group of severe peri-intraventricular haemorrhage and seven (8.5\%) in the control group (OR 7.6, 95\% Cl $2.6-20.4, p<0.001)$. Severe neurodevelopmental deficit was diagnosed in seven $(30.4 \%)$ in the severe peri-intraventricular haemorrhage group and one $(1.3 \%)$ in the control group (OR 32 ; $95 \%$ $\mathrm{Cl} 3.7$ - 281, $p<0.001)$. Individualized analysis showed that mortality was higher in peri-intraventricular haemorrhage grade III with associated cystic peri-ventricular leukomalacia (OR 4.4 95\% Cl $1.3-14.2, p=0.015)$ and in peri-intraventricular haemorrhage IV (OR 12; $95 \% \mathrm{Cl} 3.5$ - 41.2, $p<0.001$ ), when compared to controls. Differences were also noticed regarding severe neurodevelopmental deficit when compared with controls (1.3\%) in grade III peri-intraventricular haemorrhage with associated cystic peri-ventricular leukomalacia, $(75.0 \%, p<0.001)$ and grade IV peri-intraventricular haemorrhage $(50.0 \%, p<0.001)$.

Conclusion: Preterm newborns with peri-intraventricular haemorrhage grade IV or grade III with cystic peri-ventricular leukomalacia, had a higher risk of mortality and severe neurodevelopmental impairment.

Keywords: Brain/diagnostic imaging; Cerebral Intraventricular Hemorrhage; Cranial Ultrasound; Developmental Disabilities/diagnostic imaging; Infant, Very Low Birth Weight

\footnotetext{
1. Unidade de Cuidados Intensivos Neonatais. Serviço de Neonatologia B. Maternidade Bissaya Barreto. Centro Hospitalar e Universitário de Coimbra. Coimbra. Portuga $\triangle$ Autor correspondente: Adelaide Taborda. adelaide.taborda@gmail.com

Recebido: 07 de maio de 2019 - Aceite: 14 de fevereiro de 2020 - First published: 30 de outubro de 2020 - Online issue published: 03 de janeiro de 2022 Copyright @ Ordem dos Médicos 2022
} 


\section{INTRODUÇÃO}

Nas últimas duas décadas, assistiu-se a uma melhoria dos cuidados perinatais, atribuída ao uso generalizado de corticoides pré-natais, à utilização de surfactante exógeno e à transferência in utero para centros de referência, com consequente aumento da sobrevida nos recém-nascidos (RN) pré-termo (RNPT), mesmo em idades gestacionais no limiar da viabilidade. ${ }^{1-4}$ Contudo, apesar do aumento da sobrevida, a morbilidade neonatal permanece elevada, existindo um risco acrescido de complicações clínicas com repercussão no neurodesenvolvimento a curto e longo prazo, como é o caso da hemorragia peri-intraventricular (HPIV). ${ }^{3-10}$

A HPIV normalmente tem início na matriz germinativa sub-ependimária, onde se originam as futuras células neuronais e da glia, nos cérebros imaturos. ${ }^{10} \mathrm{~A}$ matriz germinativa caracteriza-se por apresentar elevada atividade metabólica, com parede endotelial nesta área ainda muito frágil e imatura. Estas vulnerabilidades associam-se à imaturidade de autorregulação cerebral, o que contribui para uma maior suscetibilidade à ocorrência de hemorragia. .,10 $^{-10}$

A ecografia transfontanelar é a técnica de neuro-imagem mais frequentemente utilizada para diagnosticar a HPIV, dado ter elevada sensibilidade, estar prontamente disponível nas unidades de cuidados intensivos neonatais e ser isenta de radiação. ${ }^{11}$

A classificação de Papile et al tem sido classicamente usada para avaliar a gravidade da hemorragia. ${ }^{12}$ Apesar de estudos recentes sugerirem a utilização de outros sistemas de classificação mais precisos, ${ }^{13}$ a classificação de Papile é ainda amplamente usada na orientação, decisão terapêutica e aconselhamento. ${ }^{14}$ A HPIV grave associa-se a maior mortalidade e alterações do neurodesenvolvimento, que incluem a paralisia cerebral (PC), défice intelectual e alte-

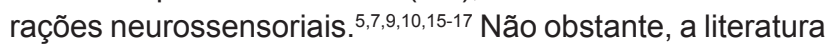
continua a ser limitada, com metodologias muito heterogéneas e amostras reduzidas devido à elevada mortalidade, mantendo-se controverso se é a HPIV em si a responsável pelas sequelas ou se estas são devidas à lesão da substância branca, que muitas vezes existe associada. Alguns estudos selecionam os doentes pelo peso de nascimento (PN), enquanto outros baseiam-se na idade gestacional (IG), e poucos são os estudos que fazem ajuste para os possíveis fatores confundidores. ${ }^{8,14,16}$ Os pais de RNPT com esta morbilidade questionam os neonatologistas, na fase aguda da doença, não só acerca da sobrevida, mas também sobre a possibilidade de sequelas graves, e daí a importância de uma melhor definição deste prognóstico.

O objetivo deste trabalho foi avaliar a mortalidade e o neurodesenvolvimento aos 24 meses de idade dos RNPT com HPIV grave, com ajuste para os cofactores que possam influenciar os resultados, e procurar avaliar individualmente o impacto da HPIV grau IV e do grau III, com ou sem atingimento da substância branca.

\section{MATERIAL E MÉTODOS}

Estudo de coorte retrospetiva, realizado numa popula- ção de RNPT internados na Unidade de Cuidados intensivos da Maternidade Bissaya Barreto - Centro Hospitalar e Universitário de Coimbra. Foram selecionados retrospetivamente os RN com HPIV grave, com idade gestacional inferior a 34 semanas, internados entre janeiro de 2006 e dezembro de 2015 na Unidade de Cuidados Intensivos Neonatais. Foram igualmente selecionados dois controlos com a mesma IG, sem HPIV, internados imediatamente após os RN com HPIV grave e registados na base de dados própria da Unidade (onde são registados todos os RN internados). A seleção dos RN e a análise dos dados clínico-demográficos maternos, perinatais e do neurodesenvolvimento, foram obtidos a partir do registo da referida base de dados da Unidade, assim como dos registos da consulta disponíveis na plataforma electrónica SClínico Hospitalar (SClínico).

Os RN sem registo no SClínico da avaliação do neurodesenvolvimento foram excluídos do estudo. A existência de malformações congénitas major foi considerada também fator de exclusão.

O diagnóstico foi realizado, como é prática na Unidade, através da avaliação seriada com ecografia cerebral, por dois neonatologistas com formação específica e experientes, seguindo o protocolo do consenso nacional. ${ }^{18}$

Foi definida HPIV grave, como a ocorrência de HPIV de graus III e IV, de acordo com classificação de Papile, ${ }^{12}$ correspondendo a HPIV grau III a hemorragia ocupando mais de $50 \%$ do ventrículo lateral, levando habitualmente à dilatação, e o grau IV indica HPIV associada a enfarte hemorrágico na substância branca ipsilateral.

Nos dois grupos foram analisados os dados clinico-demográficos maternos, perinatais e a morbilidade neonatal.

Os RN cujo PN foi inferior ao percentil 3 para a IG, segundo as curvas de Fenton, foram considerados leves para a idade gestacional (LIG). ${ }^{20}$

A persistência do canal arterial foi avaliada por ecocardiograma de forma sistemática, de acordo com protocolo ou mediante suspeita clínica. ${ }^{21}$ Definiu-se sépsis, quando o RN apresentava clínica compatível, associada a parâmetros laboratoriais positivos (contagem de leucócitos superior a $30000 / \mathrm{mm}^{3}$ ou inferior a $5000 / \mathrm{mm}^{3}$ e proteína C reativa superior a $2 \mathrm{mg} / \mathrm{dL}$ ), com ou sem hemocultura positiva. ${ }^{22} \mathrm{~A}$ enterocolite necrosante foi classificada segundo o sistema modificado de Bell. ${ }^{23} \mathrm{~A}$ displasia broncopulmonar foi definida pela necessidade de oxigenoterapia às 36 semanas de idade pós-menstrual. ${ }^{24} \mathrm{~A}$ retinopatia da prematuridade foi classificada de acordo com a classificação internacional. ${ }^{25}$ Avaliou-se a presença de leucomalácia peri-ventricular quística (LPVQ) segundo a classificação de De Vries et al. ${ }^{26}$

Todas as crianças que nasceram com IG inferior a 32 semanas ou com PN inferior a 1500 gramas foram seguidas numa consulta de follow-up neurológico da nossa Maternidade e os dados registados no Sclínico. Para a avaliação do neurodesenvolvimento psicomotor, aos 24 meses, recorreu-se à escala The Schedule of Growing SkiIls Scale II (SGS-II), nos primeiros seis anos do estudo (52 crianças) e à escala de desenvolvimento mental de Ruth 
Griffiths (RG), realizada nos últimos quatro anos do estudo (47 crianças), procedimento atual na nossa Unidade. ${ }^{27,28}$ Estas escalas foram realizadas por uma técnica com formação adequada para a sua aplicação, sem conhecimento da presença ou ausência de HPIV, que as realiza de forma sistemática a todas as crianças de risco neurológico seguidas na consulta. A primeira (SGS-II) é um teste de rastreio, que avalia nove áreas de competências e fornece um perfil de desenvolvimento; quando duas ou mais áreas se encontram desfasadas em mais do que um intervalo de idades da folha de perfil o resultado é sugestivo de atraso significativo do desenvolvimento. A escala de desenvolvimento mental de RG avalia seis áreas de competências, sendo os resultados obtidos apresentados como quocientes (das várias subescalas e global) e por idades mentais. Os quocientes das subescalas podem ser convertidos em percentis permitindo expressar o desempenho da criança relativamente à população em geral. Optou-se neste estudo por utilizar o quociente de desenvolvimento global que traduz o resultado das várias subáreas avaliadas.

Foi considerado atraso de desenvolvimento psicomotor quando no teste de RG a criança obteve um quociente de desenvolvimento (QD) global igual ou inferior a 70, ou quando no teste SGS-II o perfil era sugestivo de atraso significativo do desenvolvimento.

O diagnóstico de PC foi estabelecido de acordo com a classificação internacional e pelo sistema de classificação da função motora global. ${ }^{29,30}$ As crianças com alguma alteração motora foram avaliadas e seguidas por equipa multidisciplinar que inclui neuropediatra. Foi aceite ausência de PC perante a inexistência de algum grau de perturbação motora aos 24 meses.

A acuidade auditiva e visual foi avaliada de um modo sistemático em consultas da especialidade.

Considerou-se défice/alteração grave do neurodesenvolvimento, quando estava presente pelo menos uma das seguintes alterações: atraso do desenvolvimento psicomotor, PC, surdez neurossensorial com necessidade de prótese auditiva ou cegueira.

Avaliou-se inicialmente o impacto da HPIV grave no neurodesenvolvimento e posteriormente a repercussão, isoladamente, da HPIV de grau IV e a da HPIV grau III com ou sem LPVQ.

Para a análise estatística recorreu-se ao SPSS versão 20. Foi feita a análise univariada pelo teste $t$-student para amostras independentes, para variáveis quantitativas e teste do qui-quadrado/Fisher para variáveis categóricas; foram calculados os odds ratio (OR) e respetivo intervalo de confiança a 95\% (IC 95\%). Nas variáveis com significado estatístico, que poderiam contribuir para alterações do neurodesenvolvimento, realizou-se o ajustamento pela regressão logística. Foi considerado um valor de $p<0,05$ para indicar diferenças com significado estatístico.

Dado que se tratou de um estudo retrospectivo e com a análise dos dados anónima, não foi considerada essencial a aprovação pela Comissão de Ética. No entanto todos os procedimentos foram realizados de acordo com os regula- mentos estabelecidos pelos responsáveis da Comissão de Investigação Clínica e Ética e de acordo com a Declaração de Helsínquia da Associação Médica Mundial.

\section{RESULTADOS}

No período de estudo foram internados $1004 \mathrm{RN}$ com IG inferior a 34 semanas, tendo ocorrido $127(13 \%)$ casos de HPIV, 41 de HPIV grave (4\%): 24 de grau III $(2,4 \%)$ e 17 de grau IV $(1,7 \%)$.

No mesmo período, houve $538 \mathrm{RN}$ de muito baixo peso. A incidência de HPIV nestes grupos foram 114/538 (21\%) e da HPIV grave $38 / 538$ (7\%).

Foram avaliados $129 \mathrm{RN}$, sendo excluídos seis por ausência de registo de avaliação do neurodesenvolvimento no SClínico, e incluídos no estudo $123 \mathrm{RN}$, sendo $41 \mathrm{RN}$ com HPIV grave e 82 controlos (Fig. 1).

A média da IG foi de 27 semanas, nos dois grupos, e a média do PN foi de $1077 \mathrm{~g}$ nos RN com HPIV grave e $991 \mathrm{~g}$ nos controlos (Tabela 1).

Comparando os RN com HPIV grave com os controlos em relação às características perinatais, verificou-se que os primeiros pertencem mais ao género masculino $(73,2 \%$ vs $45,1 \%, p=0,003)$, tiveram uma utilização de corticóides pré-natais significativamente inferior $(70,7 \%$ vs $92,5 \%, p=$ $0,003)$, nasceram mais fora (outborn) da instituição $(31,7 \%$ vs $9,8 \%, p=0,002$ ) e tiveram maior taxa de reanimação com necessidade de ventilação por pressão positiva e posterior intubação endotraqueal e ventilação mecânica $(75,6 \%$ vs $48,8 \%, p=0,005)$. Os fatores de morbilidade neonatal que mostraram diferenças significativas foram a hipotensão $(36,6 \%$ vs $12,2 \%, p=0,002)$ e a sépsis neonatal $(53,7 \%$ vs $28,0 \%, p=0,005)$, respetivamente nos RN com HPIV grave em relação aos controlos. Foram incluídos no modelo de regressão logística as seguintes variáveis, que podem influenciar o neurodesenvolvimento: corticoides pré-natais, género, outborn, sépsis e hipotensão. Os corticoides pré-natais revelaram efeito protetor e apenas se mantiveram como fatores de risco independentes o género masculino, a sépsis neonatal e a hipotensão (Tabela 1).

Ocorreu LPVQ em 11 casos de HPIV grave (26,8\%): cinco $(29,4 \%)$ HPIV grau IV e seis $(25,0 \%)$ HPIV grau III (Tabela 1). Ocorreram 23 óbitos, $16(39,0 \%)$ nas HPIV graves e sete (8,5\%) nos controlos (OR 7,6; IC95\% 2,6 - 20,4; $p<0,001$ ).

Foram encontrados 11 casos de hidrocefalia, por dilatação pós-hemorrágica, dos quais sete foram submetidos a intervenção cirúrgica para drenagem e quatro não necessitaram de qualquer intervenção. Nos RN que necessitaram de alguma intervenção (reservatório ventricular ou derivação ventrículo peritoneal) ocorreu défice grave do neurodesenvolvimento em quatro, contrariamente aos RN sem necessidade de intervenção, onde não verificaram alterações graves do neurodesenvolvimento. Contudo estas diferenças não se revelaram estatisticamente significativas (Tabela 2).

Obteve-se o resultado do follow-up aos 24 meses em 99 crianças ( $99 \%$ dos sobreviventes). 
A avaliação do neurodesenvolvimento encontra-se representada na Tabela 3. A HPIV grave associou-se mais a défice grave do neurodesenvolvimento do que os controlos, respetivamente $30,4 \%$ vs 1,3\%, ( $p<0,001)$. Verificou-se maior percentagem de atraso global do DPM, em relação aos controlos, respetivamente $12,5 \%$ vs $1,3 \%(p=0,043)$ e ocorreu PC em seis crianças, todas no grupo das HPIV graves $(p<0,001)$. A classificação da função motora global foi de grau I-II em quatro crianças e grau III-IV em duas. Foi diagnosticado apenas um caso de surdez com necessidade de prótese auditiva num controlo e não se registou nenhum caso de cegueira.

A análise individualizada das HPIV graves revelou que a taxa de óbitos foi significativamente superior, quer no grau III (OR 4,4; IC 95\% 1,3 - 14,2; $p=0,015)$, quer no grau IV (OR 12; IC 95\% 3,5 - 41,2; $p<0,001)$, em relação aos controlos (Tabela 4).

Os resultados do neurodesenvolvimento das crianças com HPIV grau IV e grau III, associado ou não a lesão da substância branca, encontram-se na Tabela 4.

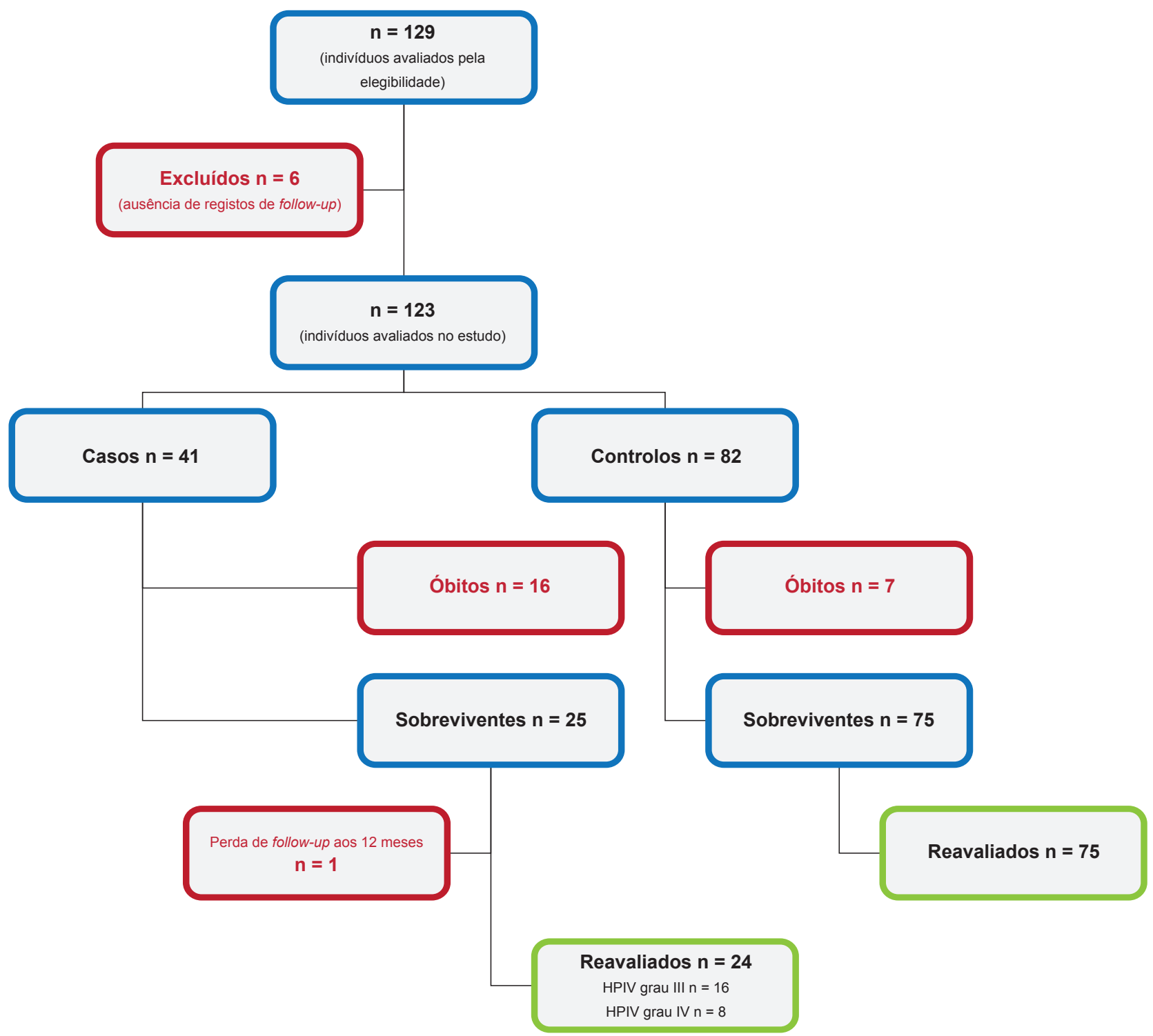

Figura 1 - Fluxograma dos recém-nascidos incluídos no estudo
Óbito ou défice grave do neurodesenvolvimento foram significativamente superiores nos RN com HPIV grau IV ou com HPIV grau III com LPVQ associada, em relação aos controlos, contrariamente à HPIV grau III sem LPVQ, que não mostrou diferenças no neurodesenvolvimento.

\section{DISCUSSÃO}

A taxa global de HPIV e da HPIV grave nos RN de muito baixo peso foi de $21 \%$ e $7 \%$ respetivamente, sobreponível à da literatura. ${ }^{1,3,17,31,32}$

Dadas as características do estudo, emparelhado pela IG, foi possível avaliar outros potenciais fatores de risco para HPIV. Identificou-se como fator de risco, após regressão logística, o género masculino, a ausência de corticoterapia pré-natal, a sépsis neonatal e a hipotensão com necessidade de inotrópicos, tal como referido na literatura. ${ }^{10,17}$

O mecanismo pelo qual os corticoides antenatais têm um efeito protetor na redução do risco de HPIV pode dever-se a uma ação multifatorial: a aceleração da maturidade pulmonar, com consequente diminuição dos distúrbios 
Tabela 1 - Características clínicas e demográficas dos recém-nascidos com hemorragia peri-intraventricular grave e dos respetivos controlos

\begin{tabular}{|c|c|c|c|c|c|}
\hline $\begin{array}{c}\text { Total } \\
(n=123)\end{array}$ & $\begin{array}{l}\text { RN com HPIV grau III-IV } \\
(n=41)\end{array}$ & $\begin{array}{l}\text { Grupo Controlo } \\
(n=82)\end{array}$ & $p$ & $\begin{array}{c}\text { OR } \\
\text { (IC } 95 \%)\end{array}$ & $\begin{array}{c}\text { ORa } \\
\text { (IC } 95 \%)\end{array}$ \\
\hline \multicolumn{6}{|l|}{ Características maternas } \\
\hline $\begin{array}{l}\text { Idade materna } \\
\text { (anos) [média (dp)] }\end{array}$ & $29,2(4,9)$ & $31,2(5.5)$ & $0,056^{*}$ & - & - \\
\hline $\begin{array}{l}\text { Primípara } \\
\mathrm{n}(\%)\end{array}$ & $22(53,7)$ & $48(58,5)$ & $0,61 £$ & - & - \\
\hline $\begin{array}{l}\text { Corioamnionite } \\
\mathrm{n}(\%)\end{array}$ & $5(12,2)$ & $8(9,80)$ & $0,758 \dagger$ & - & - \\
\hline $\begin{array}{l}\text { Pré-eclâmpsia/HTA } \\
\mathrm{n}(\%)\end{array}$ & $7(17,1)$ & $17(20,7)$ & $0,63 \dagger$ & - & - \\
\hline Nível educacional & & & & & \\
\hline $\begin{array}{l}\text { Básica } \\
\text { n (\%) }\end{array}$ & $8(24,3)$ & $15(19,2)$ & & - & - \\
\hline $\begin{array}{l}\text { Secundária } \\
\mathrm{n}(\%)\end{array}$ & $11(33,3)$ & $37(47,4)$ & & - & - \\
\hline $\begin{array}{l}\text { Universitária } \\
\mathrm{n}(\%)\end{array}$ & $14(42,4)$ & $26(33,3)$ & & - & - \\
\hline \multicolumn{6}{|l|}{ Características perinatais } \\
\hline $\begin{array}{l}\text { Parto - cesariana } \\
\mathrm{n}(\%)\end{array}$ & $23(56,1)$ & $45(54,95)$ & $0,80 £$ & - & - \\
\hline $\begin{array}{l}\text { Corticoides pré-natais } \\
\mathrm{n}(\%)\end{array}$ & $29(70,7)$ & $75(91,5)$ & $0,003 £$ & $0.22(0,1-0,6)$ & $0,26(0,1-0,9)$ \\
\hline $\begin{array}{l}\text { Índice Apgar < } 7 \text { ao } 5 \text { min } \\
\mathrm{n}(\%)\end{array}$ & $9(23,1)$ & $8(10,0)$ & $0,056 \dagger$ & - & - \\
\hline $\begin{array}{l}\text { Reanimação profunda (TET) } \\
\mathrm{n}(\%)\end{array}$ & $31(75,6)$ & $40(48,8)$ & $0,005 £$ & $3.2(1,4-7,6)$ & - \\
\hline \multicolumn{6}{|l|}{ Características neonatais } \\
\hline $\begin{array}{l}\text { Masculino } \\
\mathrm{n}(\%)\end{array}$ & $30(73,2)$ & $37(45,1)$ & $0,003 £$ & $3.3(1,4-7,5)$ & $4.7(1,6-13,9)$ \\
\hline $\begin{array}{l}\text { IG, semanas } \\
{[\text { média }(\mathrm{dp})]}\end{array}$ & $27,3(2,2)$ & $27,4(2,1)$ & $>0,99^{*}$ & - & - \\
\hline $\begin{array}{l}\mathrm{PN}, \text { gramas } \\
\text { [média }(\mathrm{dp})]\end{array}$ & 1077 (315) & $991(306)$ & $0,257^{*}$ & - & \\
\hline $\begin{array}{l}\text { Outborn } \\
\mathrm{n}(\%)\end{array}$ & $13(31,7)$ & $8(9,8)$ & $0,002 \dagger$ & $4,2(1,6-11,4)$ & - \\
\hline $\begin{array}{l}\text { LIG } \\
\mathrm{n}(\%)\end{array}$ & $4(9,8)$ & $15(18,3)$ & $0,218 \dagger$ & - & - \\
\hline $\begin{array}{l}\text { Gemelaridade } \\
\mathrm{n}(\%)\end{array}$ & $8(20,0)$ & $21(25,6)$ & $0,45 \dagger$ & & \\
\hline $\begin{array}{l}\mathrm{DMH} \\
\mathrm{n}(\%)\end{array}$ & $24(58,5)$ & $47(57,5)$ & $0,89 £$ & - & - \\
\hline $\begin{array}{l}\text { Sépsis tardia } \\
\mathrm{n}(\%)\end{array}$ & $16(39,0)$ & $16(19,8)$ & $0,022 £$ & $2,6(1,1-5,9)$ & $2,8(1,03-7,9)$ \\
\hline $\begin{array}{l}\text { DBP } \\
\text { n }(\%)\end{array}$ & $3(7,3)$ & $6(7,9)$ & $0,90 \dagger$ & & \\
\hline $\begin{array}{l}\text { PCA tratado } \\
\mathrm{n}(\%)\end{array}$ & $11(26,8)$ & $13(16,0)$ & $0,15 £$ & - & - \\
\hline $\begin{array}{l}\text { Hipotensão } \\
\text { n (\%) }\end{array}$ & $15(36,6)$ & $10(12,2)$ & $0,002 £$ & $4,1(1,6-10,3)$ & $8,3(1,8-37,6)$ \\
\hline $\begin{array}{l}\text { ENC } \\
\mathrm{n}(\%)\end{array}$ & $6(14,6)$ & $8(9,9)$ & $0.54 \dagger$ & - & \\
\hline $\begin{array}{l}\mathrm{ROP} \geq \text { grau } 3 \\
\mathrm{n}(\%)\end{array}$ & 0 & $1(1,3)$ & $0,46+$ & - & - \\
\hline $\begin{array}{l}\text { CRIB > } 5 \\
\mathrm{n}(\%)\end{array}$ & $19(46,3)$ & $23(28,0)$ & $0,044 £$ & $2,2(1,1-4,8)$ & - \\
\hline $\begin{array}{l}\text { LPVQ } \\
\mathrm{n}(\%)\end{array}$ & $11(26,8)$ & 0 & $<0,0001 \dagger$ & & \\
\hline
\end{tabular}

CRIB: clinical risk index for babies; dp: desvio padrão; DBP: displasia broncopulmonar; DMH: doença de membrana hialina; ENC: enterocolite necrosante; HPIV: hemorragia peri-intraventricular; HTA: hipertensão arterial; IC: intervalo de confiança; IG: idade gestacional, LIG: leve para idade gestacional; LPVQ: leucomalácea periventricular quística; OR: odds ratio; PCA; persistência canal arterial; PN: peso de nascimento; RN: recém-nascido; ROP: retinopatia da prematuridade; TET: tubo endotraqueal; £: qui-quadrado; †: teste de Fisher; * $\mathrm{t}$-Student para amostras independentes, com nível de significância $p<0,05$; ORa: odds ratio ajustado. 
Tabela 2 - Neurodesenvolvimento das crianças com diagnóstico de hidrocefalia

\begin{tabular}{|c|c|c|c|}
\hline & $\begin{array}{l}\text { RN com hidrocefalia com derivação } \\
\qquad n=7\end{array}$ & $\begin{array}{l}\text { RN com hidrocefalia sem derivação } \\
n=4\end{array}$ & $p$ \\
\hline $\begin{array}{l}\text { ADPM moderado a grave } \\
\mathrm{n}(\%)\end{array}$ & $2(28,6)$ & 0 & $0,49 \dagger$ \\
\hline $\begin{array}{l}\text { Paralisia cerebral } \\
\mathrm{n}(\%)\end{array}$ & $4(42,9)$ & 0 & $0,23 \dagger$ \\
\hline $\begin{array}{l}\text { Défice neurossensorial } \\
\mathrm{n}(\%)\end{array}$ & 0 & 0 & \\
\hline $\begin{array}{l}\text { Défice grave } \\
\mathrm{n}(\%)\end{array}$ & $5(57,0)$ & 0 & $0,19 \dagger$ \\
\hline
\end{tabular}

ADPM: atraso de desenvolvimento psicomotor; RN: recém-nascido; †: teste de Fisher, com nível de significância $p<0,05$

Tabela 3 - Neurodesenvolvimento das crianças com hemorragia peri-intraventricular grave aos 24 meses

\begin{tabular}{|c|c|c|c|c|}
\hline & $\begin{array}{c}\text { RN com HPIV III-IV } \\
n=24\end{array}$ & $\begin{array}{c}\text { Grupo controlo } \\
n=75\end{array}$ & $p$ & $\begin{array}{c}\text { OR } \\
\text { (IC } 95 \%)\end{array}$ \\
\hline $\begin{array}{l}\text { ADPM moderado a grave } \\
\mathrm{n}(\%)\end{array}$ & $3(12,5)$ & $1(1,3)$ & $0,043 \dagger$ & $10,5(1,1-106)$ \\
\hline $\begin{array}{l}\text { Paralisia cerebral } \\
\mathrm{n}(\%)\end{array}$ & $6(25,0)$ & 0 & $0,001 \dagger$ & \\
\hline $\begin{array}{l}\text { Défice neurosensorial } \\
\mathrm{n}(\%)\end{array}$ & 0 & $1(1,3)$ & $0,56 \dagger$ & \\
\hline $\begin{array}{l}\text { Défice grave } \\
\mathrm{n}(\%)\end{array}$ & $7(30,4)$ & $1(1,3)$ & $0,001 \dagger$ & $32(3,7-281)$ \\
\hline $\begin{array}{l}\text { Défice grave/morte }\left(^{*}\right) \\
\mathrm{n}(\%)\end{array}$ & $23(57,5)$ & $8(9,8)$ & $<0,001 \dagger$ & $12,5(4,7-32,7)$ \\
\hline
\end{tabular}

ADPM: atraso de desenvolvimento psicomotor; IC: intervalo de confiança; OR: odds ratio; RN: recém-nascido $\left(^{*}\right)$ Taxas relacionadas com o N total, que inclui óbitos; † teste de Fisher com nível de significância $p<0,05$

respiratórios, promovendo assim maior estabilidade no fluxo sanguíneo cerebral, e a estimulação da maturação da microvasculatura da matriz germinativa, são algumas das razões apontadas. ${ }^{33}$

Como descrito noutros estudos, o género masculino revelou-se um fator de risco para HPIV. ${ }^{34}$ Esta associação tem sido atribuída a melhor maturação neurovascular e aos mecanismos de regulação cerebral no género feminino. Os estrogénios foram associados a redução da lesão cerebral in vivo e in vitro, e a progesterona mostrou proteção contra lesões isquémicas ou traumáticas em modelos animais. ${ }^{35-37}$

A sépsis é um factor de risco reconhecido para HPIV devido a libertação de citoquinas vasoactivas cerebrais, ${ }^{38,39}$ tendo sido identificada como fator de risco independente neste estudo. As citoquinas libertadas podem causar alterações hemodinâmicas no endotélio vascular da matriz germinativa, ${ }^{10}$ e a instabilidade hemodinâmica, acidose metabólica e distúrbios de coagulação, num contexto de sépsis, podem levar à lesão do endotélio vascular, já por si imaturo e frágil, da matriz germinativa.

A hipotensão, ao provocar diminuição do fluxo cerebral, pode lesar os capilares da matriz germinativa por reperfusão, tendo esta associação sido reportada não só neste trabalho, como também noutros estudos, ${ }^{39}$ como fator de risco para HPIV.

Segundo a maioria dos autores, os RN com dilatação ventricular pós-hemorrágica associam-se a pior prognóstico. ${ }^{7,40,41}$ A necessidade de intervenção por dilatação ventricular tem mais sequelas do que os RN cuja dilatação estabilizou sem necessidade de cirurgia. ${ }^{7,42}$ No presente estudo, embora a percentagem de défice grave do neurodesenvolvimento fosse maior no grupo que necessitou de intervenção, não se evidenciou diferença estatisticamente significativa, provavelmente relacionado com o número reduzido de casos.

Este estudo demonstrou que os RN com HPIV grave tiveram pior prognóstico, maior mortalidade/défice grave do neurodesenvolvimento, $(57,5 \%$ vs $9,8 \%, p<0,001)$ em relação aos controlos. A elevada mortalidade dos RN com HPIV de grau IV $(52,9 \%)$ esteve de acordo com a referida noutros estudos. $9,15,16,31$

Os sobreviventes com HPIV grave tiveram mais sequelas graves do neurodesenvolvimento, nomeadamente maior taxa de PC e de atraso do desenvolvimento psico-motor (DPM). Estes resultados estão de acordo com o referido por outros autores, ${ }^{14}$ contudo, a alta taxa de mortalidade, transversal a todos os estudos, dificulta a realização de estudos de neurodesenvolvimento, pelo limitado número de sobreviventes.

A maioria dos estudos avaliam as hemorragias graves em conjunto, e por vezes, ainda, com lesões parenquimatosas associadas, ${ }^{31}$ o que limita o conhecimento sobre o real impacto das hemorragias grau III e IV isoladamente no prognóstico a curto e longo prazo.

A avaliação isolada da HPIV grau IV e grau III, com ou sem lesão parenquimatosa, mostrou que os RN com HPIV de grau III sem LPVQ não apresentavam diferença significativa, quer na PC, quer no desenvolvimento psicomotor, tal como demonstrado por O'shea et al, ${ }^{8}$ que refere que os défices associados à HPIV poderão refletir a associação 


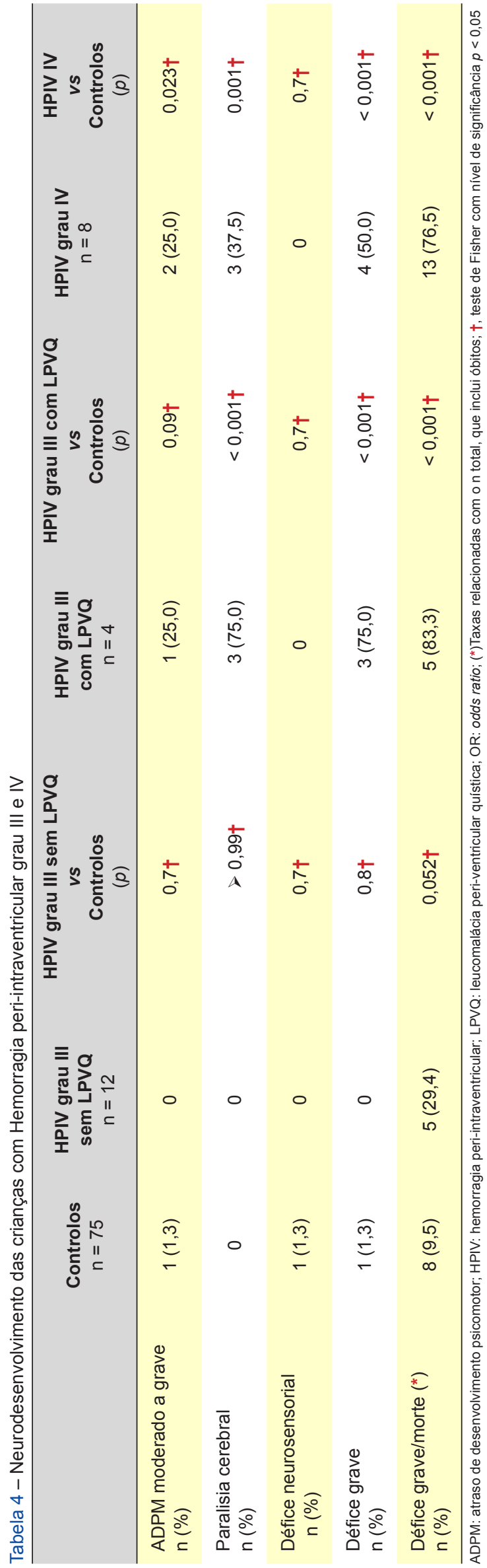

desta com lesão da substância branca.

Cerca de $80 \%$ dos RN com HPIV de grau IV faleceram ou tiveram défice grave. Três dos oito RN que sobreviveram tiveram $\mathrm{PC}(37,5 \%)$, resultados semelhantes à literatura. O atraso de DPM ocorreu em (2/8) 25\% dos RN. O pequeno número de sobreviventes com HPIV de grau IV é uma limitação do nosso estudo, contudo é uma dificuldade atual referida pela maior parte dos autores. ${ }^{43}$

Uma limitação deste estudo diz respeito ao facto de se tratar de uma análise retrospectiva. Contudo, o registo rigoroso dos RN numa base de dados da Unidade e a avaliação sistemática destas crianças em consulta, permite reduzir algumas dificuldades, habitualmente relacionadas com este tipo de estudo, tal como as frequentes falhas de informação. A avaliação do neurodesenvolvimento, numa idade precoce (24 meses) e a utilização, numa proporção significativa de casos, do teste de SGS-II (teste de rastreio), constituem outra limitação. Contudo, a ausência neste teste de alterações significativas aos 24 meses colocará como pouco provável a presença de sequelas graves. Neste estudo, poucos casos realizaram ressonância magnética (RM), a qual pode detetar lesões da substância branca e do cerebelo não objetiváveis na ecografia, o que é outra limitação a considerar.

É ponto forte deste estudo a seleção de RN com HPIV grave e controlos emparelhados pela IG. É conhecido o efeito da prematuridade na ocorrência de alterações do neurodesenvolvimento e este tipo de emparelhamento terá permitido avaliar melhor o efeito atribuído à hemorragia. Outro ponto forte a realçar refere-se à avaliação isolada da repercussão no neurodesenvolvimento dos vários tipos de HPIV grave (grau III com e sem LPVQ e grau IV). A avaliação do impacto das hemorragias grau III (com ou sem lesão da substância branca) e grau IV, separadamente, permite dar um contributo para uma melhor informação a dar aos pais, apesar de um possível subdiagnóstico das lesões difusas parenquimatosas, apenas diagnosticáveis pela RM.

Nas unidades de Cuidados Intensivos Neonatais, a definição do prognóstico dos grandes prematuros com HPIV grave é muito complexa, o que constitui uma grande preocupação quer dos profissionais de saúde, quer dos pais. ${ }^{44,45}$ Saber se o filho vai sobreviver ou se vai andar autonomamente e ter uma vida normal são as questões mais imediatas de todos os pais, às quais é difícil responder, sobretudo quando os RN associam vários fatores de risco para alterações do neurodesenvolvimento. Os resultados deste estudo representam a realidade de uma unidade de Cuidados Intensivos Neonatais, podendo contribuir para o conhecimento desta problemática, noutras unidades nacionais semelhantes.

\section{CONCLUSÃO}

O presente estudo revelou maior mortalidade e sequelas graves do neurodesenvolvimento nas crianças com HPIV de grau IV ou grau III com LPVQ, podendo assim dar algum contributo para a informação que os neonatologistas e outros técnicos possam dar os pais de RN com esta 
patologia, na fase aguda da doença.

Salienta-se, a importância da realização de um estudo multicêntrico, prospetivo e de seguimento a longo prazo destas crianças, para a avaliação do real impacto das HPIV graves, com e sem envolvimento parenquimatoso, no seu neurodesenvolvimento.

\section{CONTRIBUTO DOS AUTORES}

JA, SP: Contribuição intelectual, análise de dados, participação na escrita do manuscrito.

DF: Contribuição intelectual, revisão do manuscrito.

CR, AT: Contribuição intelectual, participação na escrita e revisão do manuscrito.

\section{PROTEÇÃO DE PESSOAS E ANIMAIS}

Os autores declaram que os procedimentos seguidos

\section{REFERÊNCIAS}

1. Horbar JD, Badger GJ, Carpenter JH, Fanaroff AA, Kilpatrick S, LaCorte $M$, et al. Trends in mortality and morbidity for very low birth weight infants, 1991-1999. Pediatrics. 2002;110:143-51.

2. Hintz SR, Poole WK, Wright LL, Fanaroff AA, Kendrick DE, Laptook $A R$, et al. Changes in mortality and morbidities among infants born at less than 25 weeks during the post-surfactant era. Arch Dis Child Fetal Neonatal Ed. 2005;90:F128-33.

3. Stoll BJ, Hansen NI, Bell EF, Shankaran S, Laptook AR, Walsh MC, et al. Neonatal outcomes of extremely preterm infants from the NICHD Neonatal Research Network. Pediatrics. 2010;126:443-56.

4. Kenet G, Kuperman AA, Strauss T, Brenner B. Neonatal IVHmechanisms and management. Thromb Res. 2011;127:S120-2.

5. Brouwer A, Groenendaal F, van Haastert IL, Rademaker K, Hanlo P, de Vries L. Neurodevelopmental outcome of preterm infants with severe intraventricular hemorrhage and therapy for post-hemorrhagic ventricular dilatation. J Pediatr. 2008;152:648-54.

6. Bolisetty S, Dhawan A, Abdel-Latif M, Bajuk B, Stack J, Lui K, et al. Intraventricular hemorrhage and neurodevelopmental outcomes in extreme preterm infants. Pediatrics. 2014;133:55-62.

7. Brouwer AJ, Groenendaal F, Benders MJ, de Vries LS. Early and late complications of germinal matrix-intraventricular haemorrhage in the preterm infant: what is new? Neonatology. 2014;106:296-303.

8. O'Shea TM, Kuban KC, Allred EN, Paneth N, Pagano M, Dammann O, et al. Neonatal cranial ultrasound lesions and developmental delays at 2 years of age among extremely low gestational age children. Pediatrics. 2008;122:e662-9.

9. Bassan $\mathrm{H}$. Intracranial hemorrhage in the preterm infant: understanding it, preventing it. Clin Perinatol. 2009;36:737-62.

10. Ballabh P. Intraventricular hemorrhage in premature infants: mechanism of disease. Pediatr Res. 2010;67:1-8.

11. Plaisier A, Raets MM, Ecury-Goossen GM, Govaert P, Feijen-Roon M, Reiss IK, et al. Serial cranial ultrasonography or early MRI for detecting preterm brain injury? Arch Dis Child Fetal Neonatal Ed. 2015;100:F293300.

12. Papile LA, Burstein J, Burstein R, Koffler $H$. Incidence and evolution of subependymal and intraventricular hemorrhage: a study of infants with birth weights less than 1,500 gm. J Pediatr. 1978;92:529-34.

13. Volpe JJ. Germinal matrix-intraventricular hemorrhage. In: Volpe JJ, editor. Neurology of the newborn. $5^{\text {th }}$ ed. Philadelphia: Saunders Elsevier; 2008. p. 517-88.

14. Mukerji A, Shah V, Shah PS. Periventricular/Intraventricular Hemorrhage and Neurodevelopmental Outcomes: a meta-analysis. Pediatrics. 2015;136:1132-43.

15. Davis AS, Hintz SR, Goldstein RF, Ambalavanan N, Bann CM, Stoll $\mathrm{BJ}$, et al. Outcomes of extremely preterm infants following severe intracranial hemorrhage. J Perinatol. 2014;34:203-8.

16. Futagi Y, Toribe Y, Ogawa K, Suzuki Y. Neurodevelopmental outcome in children with intraventricular hemorrhage. Pediatr Neurol. 2006;34:21924.

17. Calisici E, Eras Z, Oncel MY, Oguz SS, Gokce IK, Dilmen U. Neurodevelopmental outcomes of premature infants with severe estavam de acordo com os regulamentos estabelecidos pelos responsáveis da Comissão de Investigação Clínica e Ética e de acordo com a Declaração de Helsínquia da Associação Médica Mundial actualizada em 2013.

\section{CONFIDENCIALIDADE DOS DADOS}

Os autores declaram ter seguido os protocolos do seu centro de trabalho acerca da publicação de dados.

\section{CONFLITOS DE INTERESSE}

Os autores declaram não terem fontes de financiamento nem conflitos de interesse.

\section{FONTES DE FINANCIAMENTO}

Este projeto não recebeu qualquer apoio financeiro.

intraventricular hemorrhage. $\mathrm{J}$ Matern Fetal Neonatal Med. 2015;28:2115-20.

18. Taborda A, Pereira A, Graça A, Conceição C, Faria C, Trindade C et al. Revisão do consenso de neuro-imagiologia neonatal - versão maio 2013. [consultado 2017 nov 26]. Disponível em: http://www. Iusoneonatologia.com/site/upload/consensos/2010-Neuroimagiologia. pdf.

19. The International Neonatal Network. The CRIB (clinical risk index for babies) score: a tool for assessing initial neonatal risk and comparing performance of neonatal intensive care units. Lancet. 1993;342:193-8.

20. Fenton TR, Kim JH. A systematic review and meta-analysis to revise the Fenton growth chart for preterm infants. BMC Pediatr. 2013;13:59.

21. Direç̧ão Geral da Saúde. Tratamento médico e cirúrgico do canal arterial no pré-termo. Lisboa: DGS; 2012.

22. Sociedade Portuguesa de Pediatria. Secção de Neonatologia. Consenso Clínico - Procedimento no recém-nascido com risco infeccioso. Lisboa: SPP; 2014.

23. Walsh MC, Kliegman RM. Necrotizing enterocolitis: treatment based on staging criteria. Pediatr Clin North Am. 1986;33:179-201.

24. Jobe $\mathrm{AH}$, Bancalari E. Bronchopulmonary dysplasia. Am J Respir Crit Care Med. 2001;163:1723-9.

25. International Committee for the Classification of Retinopathy of Prematurity. The International Classification of Retinopathy of Prematurity revisited. Arch Ophthalmol. 2005;123:991-9.

26. de Vries LS, Eken P, Dubowitz LM. The spectrum of leukomalacia using cranial ultrasound. Behav Brain Res. 1992;49:1-6.

27. Bellman M, Lingam S, Aukett A. SGS II Escala de avaliação das competências no desenvolvimento infantil II. $2^{a}$ ed. Lisboa: Hogrefe; 2003.

28. Luiz D, Faragher B, Barnard A, Knoesen N, Kotras N, Griffiths LB Mental developmental scales - extended revised. Oxford: Hogrefe; 2006.

29. Rosenbaum P, Paneth N, Leviton A, Goldstein M, Bax M, Damiano D, et al. A report: the definition and classification of cerebral palsy April 2006. Dev Med Child Neurol Suppl. 2007;109:8-14

30. Palisano R, Rosenbaum P, Walter S, Russell D, Wood E, Galuppi B. Development and reliability of a system to classify gross motor function in children with cerebral palsy. Dev Med Child Neurol. 1997;39:214-23.

31. Larroque B, Marret S, Ancel PY, Arnaud C, Marpeau L, Supernant K, et al. White matter damage and intraventricular hemorrhage in very preterm infants: the EPIPAGE study. J Pediatr. 2003;143:477-83.

32. Patra K, Wilson-Costello D, Taylor HG, Mercuri-Minich N, Hack M. Grades I-II intraventricular hemorrhage in extremely low birth weight infants: effects on neurodevelopment. J Pediatr. 2006;149:169-73.

33. Vinagre FE, Marba ST. Uso antenatal do corticosteroide e hemorragia peri-intraventricular. Rev Paul Pediatr. 2010;28:346-52

34. Mohamed MA, Aly $\mathrm{H}$. Male gender is associated with intraventricular hemorrhage. Pediatrics. 2010;125:e333-9.

35. Nunez JL, McCarthy MM. Sex differences and hormonal effects in a model of preterm infant brain injury. Ann N Y Acad Sci. 2003;1008:281-4.

36. Schauwecker PE, Wood RI, Lorenzana A. Neuroprotection against 
excitotoxic brain injury in mice after ovarian steroid depletion. Brain Res. 2009;1265:37-46.

37. Kadri $\mathrm{H}$, Mawla AA, Kazah J. The incidence, timing, and predisposing factors of germinal matrix and intraventricular hemorrhage $(\mathrm{GMH} / \mathrm{IVH})$ in preterm neonates. Childs Nerv Syst. 2006;22:1086-90.

38. Linder N, Haskin O, Levit O, Klinger G, Prince T, Naor N, et al. Risk factors for intraventricular hemorrhage in very low birth weight premature infants: a retrospective case-control study. Pediatrics. 2003;111:e590-5.

39. Vural M, Yilmaz I, Ilikkan B, Erginoz E, Perk Y. Intraventricular hemorrhage in preterm newborns: risk factors and results from a University Hospital in Istanbul, 8 years after. Pediatr Int. 2007;49:341-4.

40. Brouwer AJ, van Stam C, Uniken Venema M, Koopman C, Groenendaa $\mathrm{F}$, de Vries LS. Cognitive and neurological outcome at the age of 5-8 years of preterm infants with post-hemorrhagic ventricular dilatation requiring neurosurgical intervention. Neonatology. 2012;101:210-6.

41. Persson EK, Hagberg G, Uvebrant P. Hydrocephalus prevalence and outcome in a population-based cohort of children born in 1989-1998. Acta Paediatr. 2005:726-32.

42. Srinivasakumar $P$, Limbrick $D$, Munro $R$, Mercer $D$, Rao $R$, Inder $\mathrm{T}$, et al. Posthemorrhagic ventricular dilatation-impact on early neurodevelopmental outcome. Am J Perinatol. 2013;30:207-14

43. Sherlock RL, Anderson PJ, Doyle LW, Victorian Infant Collaborative Study Group. Neurodevelopmental sequelae of intraventricular haemorrhage at 8 years of age in a regional cohort of ELBW/very preterm infants. Early Hum Dev. 2005;81:909-16.

44. Jones R, Clark E, Broad K, Smit E. Outcome following preterm intraventricular haemorrhage - what to tell the parents. Pediatr Child Health. 2018;28:431-5.

45. Jeschk E, Biermann A, Gunsten C, Böhler T, Heller G, Hummler H, et al. Mortality and major morbidity of very-low-birth-weight infants in Germany 2008-2012: a report based on administrative data. Front Pediatr. 2016;4:1-8. 